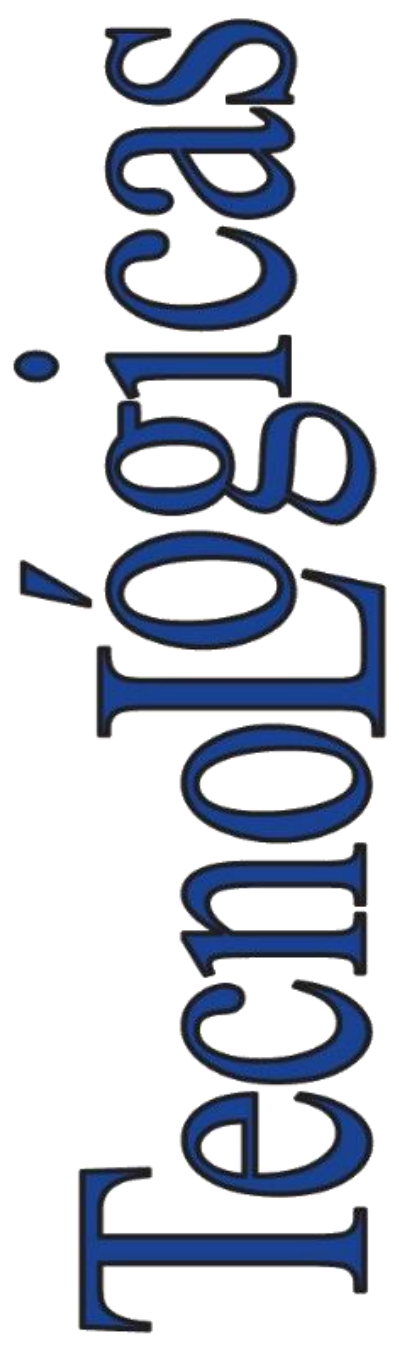

ISSN-p: 0123-7799

ISSN-e: $2256-5337$

Vol. 24, nro. 52, e2118, 2021

Recibido: 09 agosto 2021 Aceptado: 23 noviembre 2021 Disponible: 16 diciembre 2021

CInstituto Tecnológico Metropolitano Este trabajo está licenciado bajo una Licencia Internacional Creative Commons Atribución (CC BY-NC-SA)

\section{Alteración de la entropía en la precuña y la corteza cingulada posterior en la enfermedad de Alzheimer: estudio de resonancia magnética funcional en reposo}

\author{
Alteration of Entropy in the Precuneus and Posterior \\ Cingulate Cortex in Alzheimer's Disease: A Resting-State \\ Functional Magnetic Resonance Study
}

\author{
(D) Aura C. Puche'; \\ D John Fredy Ochoa-Gómez²; \\ (D) Yésika Alexandra Agudelo-Londoño ; \\ (D) Jan Karlo Rodas-Marín"; \\ (D) Carlos Andrés Tobón-Quintero 5 \\ ${ }^{1}$ Universidad de Antioquia, Medellín-Colombia, \\ aura.puche@udea.edu.co \\ 2 Universidad de Antioquia, Medellín-Colombia, \\ john.ochoa@udea.edu.co \\ ${ }^{3}$ Universidad de Antioquia, Medellín-Colombia, \\ yesika.agudelo@udea.edu.co \\ ${ }^{4}$ Institución Prestadora de Servicios de Salud "IPS Universitaria", \\ Medellín-Colombia, jan.rodas@udea.edu.co \\ 5 Institución Prestadora de Servicios de Salud "IPS Universitaria", \\ Medellín-Colombia, carlos.tobonq@udea.edu.co
}

Cómo citar / How to cite

A. C. Puche; J. F. Ochoa-Gómez; Y. A. Agudelo-Londoño; J. K. RodasMarín; C. A. Tobón-Quintero, "Alteración de la entropía en la precuña y la corteza cingulada posterior en la enfermedad de Alzheimer: estudio de resonancia magnética funcional en reposo", TecnoLógicas, vol. 24, nro. 52, e2118, 2021. https://doi.org/10.22430/22565337.2118 


\section{Resumen}

El cerebro humano ha sido descrito como un sistema complejo. Su estudio por medio de señales neurofisiológicas ha desvelado la presencia de interacciones lineales y no lineales. En este contexto, se han utilizado métricas de entropía para descubrir el comportamiento cerebral en presencia y ausencia de alteraciones neurológicas. El mapeo de la entropía es de gran interés para el estudio de enfermedades neurodegenerativas progresivas como la enfermedad de Alzheimer. El objetivo de este estudio fue caracterizar la dinámica de las oscilaciones cerebrales en dicha enfermedad por medio de la entropía y la amplitud de las oscilaciones de baja frecuencia a partir de señales Bold de la red por defecto y la red de control ejecutivo en pacientes con Alzheimer e individuos sanos, utilizando una base de datos extraída de la serie de estudios de imágenes de acceso abierto. Los resultados revelaron mayor poder discriminatorio de la entropía por permutaciones en comparación a la amplitud de fluctuación de baja frecuencia y la amplitud fraccional de fluctuaciones de baja frecuencia. Se obtuvo un incremento de la entropía por permutaciones en regiones de la red por defecto y la red de control ejecutivo en pacientes. La corteza cingulada posterior y la precuña manifestaron característica diferencial al evaluar la entropía por permutaciones en ambos grupos. No hubo hallazgos al correlacionar las métricas con las escalas clínicas. Los resultados demostraron que la entropía por permutaciones permite caracterizar la función cerebral en pacientes con Alzheimer, además revela información sobre las interacciones no lineales complementaria a las características obtenidas por medio del cálculo de la amplitud de las oscilaciones de baja frecuencia.

\section{Palabras clave}

Resonancia magnética funcional, enfermedad de Alzheimer, procesamiento de imágenes médicas, entropía por permutaciones, red por defecto, red de control ejecutivo.

\section{Abstract}

The human brain has been described as a complex system. Its study using neurophysiological signals has revealed the presence of linear and non-linear interactions. In this context, entropy metrics have been used to discover brain behavior in the presence and absence of neurological alterations. Entropy mapping is of great interest for the study of progressive neurodegenerative diseases such as Alzheimer's Disease (AD). The objective of this study was to characterize the dynamics of brain oscillations in $\mathrm{AD}$ using entropy and the Amplitude of Low-Frequency Fluctuations (ALFF) of BOLD signals from the default network and the executive control network in patients with $\mathrm{AD}$ and healthy individuals. For this purpose, the data was extracted from the Open Access Series of Imaging Studies (OASIS). The results revealed greater discriminatory power in Permutation Entropy (PE) than in ALFF and fractional ALFF metrics. An increase in PE was obtained in regions of the default network and the executive control network in patients. The posterior cingulate cortex and the precuneus exhibited a differential characteristic when PE was evaluated in both groups. There were no findings when the metrics were correlated with clinical scales. The results showed that PE can be used to characterize the brain function in patients with $\mathrm{AD}$ and reveals information about non-linear interactions complementary to the characteristics obtained by calculating the ALFF.

\section{Keywords}

Functional magnetic resonance imaging, Alzheimer's disease, Permutation entropy, Medical image processing, Default mode network, Executive network. 


\section{INTRODUCCIÓN}

La enfermedad de Alzheimer (EA) es la causa más común de demencia [1]. Según proyecciones realizadas en el 2014 [2], el número de personas con EA en Colombia para el 2020 es de 260000 con un costo aproximado de 1 billón de pesos [3]. La EA se caracteriza por una acumulación excesiva de placas de B-amiloide extracelular y la presencia de ovillos neurofibrilares de proteína tau hiperfosforilada intracelulares, que da lugar a una configuración atípica que se extiende por toda la corteza cerebral [4]. La EA ha sido descrita por tres estadios: la etapa preclínica, en el que inicia la acumulación atípica de B-amiloide sin manifestaciones clínicas; el deterioro cognitivo leve (DCL), que puede ser de tipo amnésico y no amnésico; y, por último, la etapa de demencia, en la que se identifican alteración de la memoria y falta de independencia del individuo para realizar tareas de la vida diaria. Esta última etapa, a su vez, tiene tres fases: leve, moderada y severa [5].

Distintas modalidades de imágenes han sido ampliamente usadas para el estudio de la EA. En particular, la resonancia magnética funcional es considerada una tecnología que podría tener relevancia clínica debido a su prometedor potencial para la identificación de alteraciones cerebrales causadas por la enfermedad [6]. El estudio de las oscilaciones cerebrales por medio de la resonancia magnética funcional en reposo (rs-fMRI) a través del análisis de la señal dependiente del nivel de oxigenación sanguínea (BOLD, por sus siglas en inglés: Blood-Oxygenation-Level-Dependent) ha demostrado alteraciones en la red por defecto (RD) y la red de control ejecutivo (RCE) en pacientes con EA [7]-[10].

El uso de métricas de conectividad basadas en teoría de grafos y el análisis de componentes independientes ha permitido identificar un deterioro acelerado en sujetos con EA en regiones específicas del cerebro, como es el caso de las áreas involucradas en la red por defecto, mientras que en sujetos con envejecimiento normal se han identificado disrupciones con ocurrencia no uniformemente en todo el cerebro [11]. Por otro lado, el uso de las métricas espectrales tales como la amplitud de oscilaciones de baja frecuencia (ALFF, por sus siglas en inglés: Amplitude of Low Frequency Fluctuations) y su versión fraccional (fALFF, por sus siglas en inglés: fractional ALFF) [12] han mejorado el desempeño de algoritmos de aprendizaje de máquina para la discriminación de sujetos con DCL y EA de individuos cognitivamente normales [13].

A pesar de que la rs-fMRI ha arrojado información relacionada con la dinámica cerebral, aún no es una técnica usualmente utilizada en entornos clínicos debido a factores como la presencia de artefactos en las señales y la ausencia de métricas de referencia (gold standard) para la estimación de la función cerebral [6], [14]. Por lo tanto, algunos esfuerzos se han concentrado en realizar innovaciones metodológicas que permitan comprender la función cerebral a partir del análisis de las interacciones de naturaleza no lineal que caracterizan a las señales neurofisiológicas [15]. El uso de métodos basados en teoría de la información permite capturar interacciones no lineales que pueden ser beneficiosas para el entendimiento de la función cerebral [15], además estudios recientes resaltan que el uso de enfoques no lineales para el estudio de la función cerebral brinda información relevante para el estudio en cerebros sanos y patológicos [16].

Varios enfoques se han utilizado para estimar la complejidad cerebral por medio de métricas no lineales, dentro de ellas se encuentra una variedad de enfoques para el cálculo de la entropía [17]. Un enfoque menos usado es la entropía por permutaciones, un método simple, rápido y robusto para el análisis de series de tiempo caóticas [18]. Esta métrica ha sido utilizada previamente para el análisis de la señal BOLD en sujetos de la iniciativa de Neuroimágenes de la Enfermedad de Alzheimer (ADNI, por sus siglas en inglés) con DCL, EA y controles en donde se encontró una disminución de la entropía en pacientes con EA en 
comparación con sujetos con DCL y controles. El estudio reportó hallazgos significativos de correlación entre la métrica y las escalas clínicas Mini Mental y valoración clínica de la demencia. El análisis fue realizado con señales BOLD de 130 puntos de tiempo y una dimensión embebida de 4. Los parámetros utilizados son resaltados por los autores como una limitación del estudio [19]. Hasta donde tienen conocimiento los autores, es el único estudio realizado en EA utilizando la métrica de entropía por permutaciones, lo que evidencia que a la fecha existen pocos estudios que hayan utilizado métricas de complejidad para el análisis de datos de fMRI en EA [20].

En este estudio se utilizaron datos de rs-fMRI con 164 puntos de tiempo de la iniciativa de acceso abierto OASIS-3 (en inglés: Open Access Series of Imaging Studies: Longitudinal MRI Data in Non-demented and Demented Older Adults) [21] de sujetos rotulados como cognitivamente normales $(\mathrm{CN})$ y con EA. Se cuantificó la función cerebral por medio de la entropía por permutaciones y se comparó con el ALFF y fALFF para la red por defecto y la red de control ejecutivo; adicionalmente, las métricas fueron correlacionadas con escalas clínicas. Lo anterior, con el objetivo de evaluar si el uso de la entropía por permutaciones brinda información adicional a la obtenida con métricas espectrales, sobre la dinámica de las oscilaciones cerebrales y brinda características diferenciales en pacientes con EA y sujetos sanos al ser evaluadas en las redes cerebrales en reposo que se ven mayormente afectadas en la EA.

\section{METODOLOGÍA}

\subsection{Sujetos}

Datos de resonancia magnética estructural y resonancia magnética funcional etiquetados como EA y CN fueron descargados de la base de datos OASIS-3 [21], un proyecto longitudinal en donde a varios individuos se les realizó más de una adquisición. Dos expertos analizaron la información clínica disponible en la plataforma de los sujetos etiquetados como EA y CN [21], teniendo en cuenta información como: edad, antecedentes personales, edad de inicio de los síntomas, cuadro clínico registrado en el tiempo, evolución de las alteraciones cognitivas, puntajes en pruebas neuropsicológicas y escalas de funcionalidad disponibles. A partir de la revisión se excluyeron sujetos etiquetados como EA que durante el seguimiento longitudinal tuvieron un diagnóstico posterior de $\mathrm{CN}$, cuadro clínico leve que sugería un síndrome demencial incierto, evolución de los síntomas que aparentaban una demencia debido a otra condición o enfermedad neurodegenerativa, presentar síntomas depresivos que pudieran explicar las quejas cognitivas, tener la resonancia previa al inicio de los síntomas o tener antecedente de una enfermedad neurológica mayor distinta a EA que pudiera alterar los resultados (como antecedente de enfermedad cerebrovascular o traumatismo encefalocraneano).

Luego de realizar la revisión clínica, se preprocesaron datos de 117 sujetos, de los cuales 23 fueron descartados por exceso de movimiento (EA: 12, CN:11) y 1 sujeto del grupo CN fue descartado por tener valores atípicos en varias regiones de la red por defecto. Finalmente se incluyeron datos de 93 sujetos (EA: 36, CN:57) para los análisis posteriores. Si bien la base de datos no proporciona específicamente información sobre la gravedad de la enfermedad, esta se determinó por la suma de cajas de la escala de valoración clínica de la demencia (CDRSB, por sus siglas en inglés: Dementia Rating Scale Sum of Boxes) y la escala Mini Mental (MMSE, por sus siglas en inglés: Mini-Mental State Examination). Dado que los individuos 
del grupo EA están caracterizados por un $4.5<$ CDR-SB $<9$ pertenecen a la categoría Alzheimer leve [22]. La información demográfica detallada puede observarse en la Tabla 1.

Tabla 1. Información demográfica de los sujetos de estudio. Fuente: elaboración propia.

\begin{tabular}{cccc}
\hline Parámetro & EA & CN & Valor p \\
& Media \pm Desviación estándar & Media \pm Desviación estándar & - \\
Sexo (femenino: masculino) & 36 & 57 & 0.48 \\
Edad & $16: 20$ & $24: 33$ & $<0.01$ \\
Escolaridad (años) & $74.70 \pm 5.89$ & $70.97 \pm 6.52$ & 0.72 \\
MMSE & $15.33 \pm 2.77$ & $15.68 \pm 2.70$ & $<0.01$ \\
CDR-SB & $25.19 \pm 3.14$ & $28.91 \pm 1.24$ & $<0.01$ \\
\hline
\end{tabular}

\subsection{Adquisición de las imágenes}

Las imágenes de rs-fMRI fueron adquiridas con un escáner SIEMENS 3T (MAGNETOM Trio Tim, Siemens, Erlangen, Germany). Se adquirieron 164 volúmenes de 36 cortes cada uno y tamaño de voxel de $4 \times 4 \times 4 \mathrm{~mm}$ con los parámetros: tiempo eco $(\mathrm{TE})=27 \mathrm{~ms}$, tiempo de repetición $(\mathrm{TR})=2200 \mathrm{~ms}$, ángulo de giro $(\mathrm{FA})=90^{\circ}$, tamaño de la matriz $=64$. Información adicional relacionada con la adquisición de las imágenes se puede encontrar en [23].

\subsection{Procesamiento de las imágenes}

El preprocesamiento de las imágenes fue realizado en la toolbox CONN [24]. Se hizo una inspección visual para descartar imágenes de baja calidad. Posteriormente se realizó realineación de los datos tomando el primer corte como referencia, corrección temporal y registro con la imagen $\mathrm{T} 1$ correspondiente. Luego se inspeccionó el reporte de corrección de movimiento, se excluyeron los sujetos con movimiento de cabeza $>2^{\circ}$ en rotación y $>2 \mathrm{~mm}$ en traslación en cualquier dirección. Las imágenes de los 117 sujetos fueron preprocesadas, de los cuales 23 sujetos (EA:12, CN:11) fueron descartados por exceso de movimiento.

Las imágenes de los 94 sujetos restantes fueron normalizadas al espacio estándar definido por el Instituto Neurológico de Montreal (MNI, por sus siglas en inglés: Montreal National Institute), conservando el tamaño de voxel 2x2x2 mm. Se realizó eliminación de tendencias de movimiento, señales relacionadas con la sustancia blanca y líquido cefalorraquídeo. Se aplicó un filtro pasa-banda en el rango de frecuencia de $0.01 \mathrm{~Hz}$ a $0.1 \mathrm{~Hz}$.

\subsection{Extracción de la señal BOLD}

La extracción de las señales se realizó a partir de las plantillas propuestas por [25] para la red por defecto y la red de control ejecutivo (Figura 1). Se utilizaron máscaras de cada región con número de voxeles mayor a 50, por lo tanto, se extrajeron 17 señales BOLD para la red por defecto y 11 señales BOLD para la red de control ejecutivo. El proceso de obtención de las señales fue realizado con la herramienta DPARSFA [26] (DPABI_V4.1_190725). En la Tabla 2 se dan detalles de los nombres de cada región involucrada. 


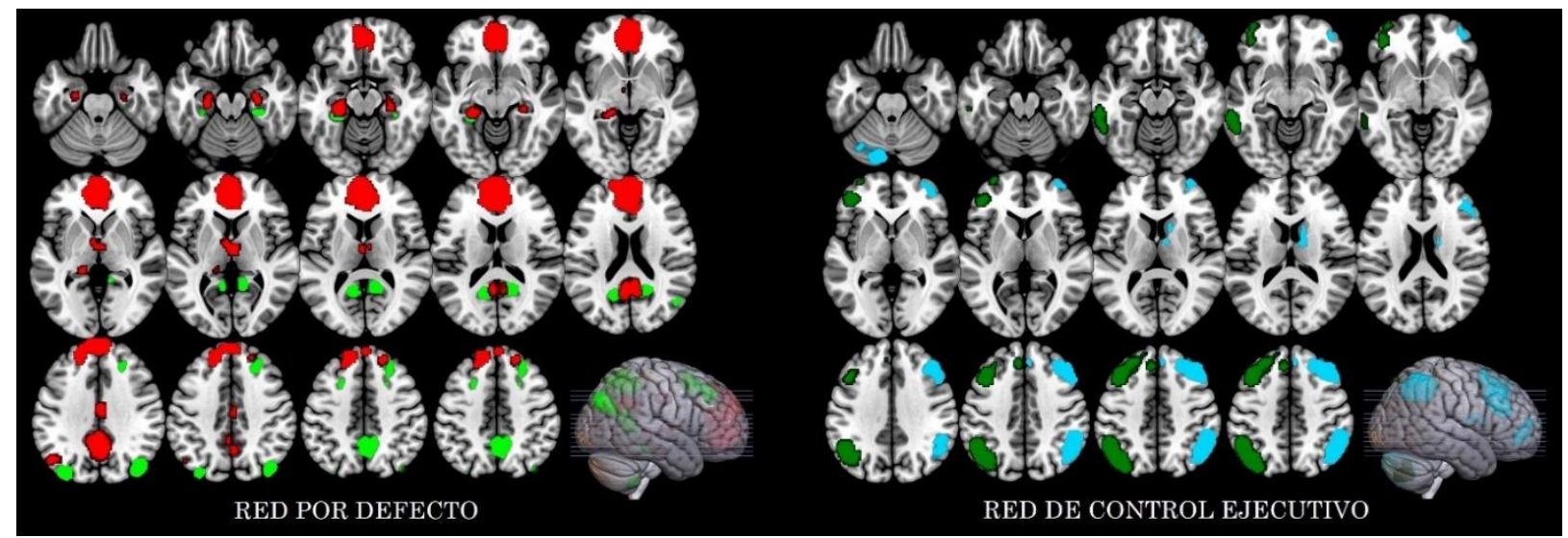

Figura 1. Regiones de interés de la red por defecto y la red de control ejecutivo. Fuente: elaboración propia.

\subsection{Análisis de la señal BOLD}

Para cada región de la RD y la RCE se aplicaron las medidas espectrales: ALFF y fALFF y la métrica no lineal: entropía por permutaciones (PE, por sus siglas en inglés: Permutation Entropy). Todas las métricas fueron calculadas sobre las señales en la banda de frecuencia de $0.01 \mathrm{~Hz}$ a $0.1 \mathrm{~Hz}$.

\subsubsection{Amplitud de las fluctuaciones de baja frecuencia}

La ALFF se define como la suma de amplitudes en una banda de frecuencia, mientras que la fALFF se define como la fracción de la suma de amplitudes en una banda de frecuencia. Para una serie de tiempo de longitud N definida en (1), el cálculo de las métricas ALFF y fALFF está representado por (2) y (3), respectivamente [27].

$$
\begin{gathered}
x(t)=\sum_{k=1}^{N}\left[a_{k} \cos \left(2 \pi f_{k} t\right)+b_{k} \sin \left(2 \pi f_{k} t\right)\right] \\
A L F F=\sum_{k: f_{k} \in[0.01,0.08]} \sqrt{\frac{a_{k}^{2}\left(f_{k}\right)+b_{k}^{2}\left(f_{k}\right)}{N}} \\
f A L F F=\frac{\sum_{k: f_{k} \in[0.01,0.08]} \sqrt{\frac{a_{k}^{2}\left(f_{k}\right)+b_{k}^{2}\left(f_{k}\right)}{N}}}{\sum_{k=1}^{N} \sqrt{\frac{a_{k}^{2}\left(f_{k}\right)+b_{k}^{2}\left(f_{k}\right)}{N}}}
\end{gathered}
$$

\subsubsection{Entropía por permutaciones}

La entropía por permutaciones es un enfoque propuesto por [18] para el análisis de series de tiempo complejas y caóticas, la cual tiene la ventaja de dar resultados significativos en presencia de ruido observacional y dinámico, además, es un método simple, de cálculo rápido y robusto. Para una serie de tiempo $\left\{x_{t}\right\}=1, \ldots, T, \pi$ permutaciones de orden $n$, la PE se define por (4), donde $p(\pi)$ está definido por (5), donde el símbolo \# hace referencia a "número" [18]. 


$$
\begin{gathered}
H(n)=-\sum p(\pi) \log (p(\pi)) \\
p(\pi)=\frac{\#\left\{t \mid t \leq T-n,\left(x_{t+1}, \ldots, x_{t+n}\right) \text { has type } \pi\right\}}{T-n+1}
\end{gathered}
$$

Tabla 2. Nombre de las regiones que conforman las redes según la plantilla de referencia

\begin{tabular}{|c|c|c|c|}
\hline Etiqueta & Red por defecto & Etiqueta & Red de Control Ejecutivo \\
\hline 1 & $\begin{array}{l}\text { Corteza Prefrontal Medial, Corteza } \\
\text { Cingulada Anterior y Corteza Orbitofrontal } \\
\text { (B) }\end{array}$ & 1 & $\begin{array}{l}\text { Giro Frontal Medio, Giro Frontal } \\
\text { Superior (I) }\end{array}$ \\
\hline 2 & Giro Angular (I) & 2 & $\begin{array}{l}\text { Giro Frontal Inferior, Giro } \\
\text { Orbitario Frontal (I) }\end{array}$ \\
\hline 3 & Giro Frontal Superior (D) & 3 & $\begin{array}{l}\text { Lóbulo Parietal Superior, Lóbulo } \\
\text { Parietal Inferior, Precuña, Giro } \\
\text { Angular (I) }\end{array}$ \\
\hline 4 & Corteza Cingulada Posterior, Precuña (B) & 4 & $\begin{array}{l}\text { Giro Temporal Inferior, Giro } \\
\text { Temporal Medio (I) }\end{array}$ \\
\hline 5 & Corteza Cingulada Anterior Dorsal (B) & 5 & Crus I (D) \\
\hline 6 & Tálamo (B) & 6 & $\begin{array}{l}\text { Giro Frontal Medio, Giro Frontal } \\
\text { Superior (D) }\end{array}$ \\
\hline 7 & Hipocampo (I) & 7 & Giro Frontal Medio (D) \\
\hline 8 & Hipocampo (D) & 8 & $\begin{array}{l}\text { Lóbulo Parietal Inferior, Giro } \\
\text { Supramarginal, Giro Angular (D) }\end{array}$ \\
\hline 9 & $\begin{array}{l}\text { Corteza Retrospenial, Corteza Cingulada } \\
\text { Posterior (I) }\end{array}$ & 9 & Giro Frontal Superior (D) \\
\hline 10 & Giro Frontal Medio (I) & 10 & Crus I, Crus II, Lóbulo VI (D) \\
\hline 11 & Giro Parahipocampal (I) & 11 & Núcleo Caudado (D) \\
\hline 12 & Giro Occipital Medio (I) & - & - \\
\hline 13 & $\begin{array}{l}\text { Corteza Retrospenial, Corteza Cingulada } \\
\text { Posterior (D) }\end{array}$ & - & - \\
\hline 14 & Precuña (B) & - & - \\
\hline 15 & $\begin{array}{l}\text { Giro Frontal Superior, Giro Frontal Medio } \\
\text { (D) }\end{array}$ & - & - \\
\hline 16 & Giro Parahipocampal (D) & - & - \\
\hline 17 & Giro Angular. Giro Occipital Medio (D) & - & - \\
\hline
\end{tabular}
Fuente: elaboración propia.

* D: regiones ubicadas en el hemisferio derecho, I: regiones ubicadas en el hemisferio izquierdo y B: regiones localizadas en ambos hemisferios.

Para el cálculo de la EP se utilizó un retraso embebido $(l)$ de (2) y una dimensión embebida ( $m$ ) de (4) [28], donde $m$ y $l$ cumplieron la condición definida por (6) para evitar el submuestreo de las señales.

$$
m ! \leq N-(m-1)
$$


$N=164$, corresponde a la longitud de la serie de tiempo. El parámetro $l$ no tiene mucha influencia en la entropía de la serie de tiempo, su valor se tomó con base en la literatura reportada por estudios de electroencefalografía [28]. La medida fue estandarizada por el algoritmo natural de $m$ !, donde PE alcanza su valor máximo, (7):

$$
P E=\frac{H(n)}{\ln (m !)}
$$

\subsubsection{Análisis estadístico}

Se removió el efecto de la edad de las métricas por medio de una regresión lineal. Para la comparación entre grupos se utilizó una prueba estadística no paramétrica de dos muestras, descrita por [29], con nivel de significancia $p<0.05$ y se realizó corrección por medio del método (FDR, por sus siglas en inglés: False Discovery Rate, p_fdr). Se analizaron los valoresp sin corrección (p_unc) para comparaciones múltiples por tratarse de un estudio exploratorio sobre la métrica PE y por ser un estudio con tamaño de muestra diferente entre los grupos (CN:57, AD:36). Se calculó el tamaño del efecto con la g de Hedges, utilizando la herramienta The Measures of Effect Size Toolbox [30]; los hallazgos se discuten principalmente con esta medida. Adicionalmente, se realizó un análisis de correlación utilizando el índice de correlación de Spearman entre las escalas de valoración clínica MMSE, CDR-SB y las métricas obtenidas en el análisis de la señal BOLD del grupo EA.

Las métricas se llevaron al rango de 0 a 1 para mejorar la visualización de los resultados por medio de diagramas de cajas.

\section{RESULTADOS}

\subsection{Red por defecto}

Se observó un incremento estadísticamente significativo en la entropía del grupo EA en comparación con el grupo $\mathrm{CN}$ en la región 10 que involucra a la corteza retrosplenial y la corteza cingulada posterior del hemisferio izquierdo, con tamaño del efecto mediano de valor -0.57. El incremento en la entropía también se pudo observar en la región 4, que involucra la corteza cingulada posterior y precuña, con tamaño del efecto mediano de valor -0.57 (Tabla 3 y Figura 2).

No se observaron diferencias entre grupos para las métricas ALFF y fALFF, el diagrama de cajas puede visualizarse en las Figuras S1 y S2 del apéndice. Los valores obtenidos al correlacionar las métricas con las escalas clínicas CDR-SB y MMSE para las regiones de la red por defecto se encuentran en las Tablas S1 y S2, respectivamente del apéndice. 
Tabla 3. Valores-p y tamaño del efecto para las 17 regiones de la red por defecto Fuente: elaboración propia.

\begin{tabular}{|c|c|c|c|c|c|c|c|c|c|}
\hline \multirow{2}{*}{ ROI } & \multicolumn{3}{|c|}{$\mathrm{ALFF}$} & \multicolumn{3}{|c|}{ fALFF } & \multicolumn{3}{|c|}{$\mathrm{PE}$} \\
\hline & p1_unc & p2_unc & ES & p1_unc & p2_unc & ES & p1_unc & p2_unc & ES \\
\hline 1 & .40 & .60 & -.01 & .43 & .57 & .05 & .21 & .79 & .15 \\
\hline 2 & .29 & .71 & .08 & .09 & .91 & .16 & .08 & .92 & .35 \\
\hline 3 & .47 & .53 & -.03 & .72 & .28 & -.08 & .54 & .46 & .01 \\
\hline 4 & .47 & .53 & -.04 & .46 & .54 & -.07 & .99 & .01 & -.57 \\
\hline 5 & .53 & .47 & -.05 & .77 & .23 & -.16 & .47 & .53 & .06 \\
\hline 6 & .88 & .12 & -.29 & .70 & .30 & -.10 & .24 & .76 & .08 \\
\hline 7 & .85 & .15 & -.32 & .45 & .55 & .03 & .08 & .92 & .37 \\
\hline 8 & .50 & .50 & -.12 & .27 & .73 & .12 & .18 & .82 & .29 \\
\hline 9 & .45 & .55 & -.02 & .51 & .49 & -.01 & 1.0 & $.00 *$ & -.57 \\
\hline 10 & .40 & .60 & -.03 & .65 & .35 & -.12 & .59 & .41 & -.04 \\
\hline 11 & .86 & .14 & -.28 & .77 & .23 & -.09 & .84 & .16 & -.19 \\
\hline 12 & .26 & .74 & .09 & .36 & .64 & .07 & .16 & .84 & .26 \\
\hline 13 & .59 & .41 & -.13 & .52 & .48 & -.04 & .81 & .19 & -.07 \\
\hline 14 & .29 & .71 & .09 & .80 & .20 & -.25 & .87 & .13 & -.28 \\
\hline 15 & .44 & .56 & -.07 & .53 & .47 & .00 & .87 & .13 & -.23 \\
\hline 16 & .82 & .18 & -.25 & .83 & .17 & -.19 & .58 & .42 & .00 \\
\hline 17 & .37 & .63 & .02 & .59 & .41 & -.12 & .55 & .45 & .06 \\
\hline
\end{tabular}

p1_unc: valor-p no corregido para la cola $C N>A D$, p2_unc: valor-p no corregido para la cola $C N<A D$, ES: tamaño del efecto, I: izquierdo, D: derecho, *: región con $p \_f d r<0.05$ ( $\left.p 1 \_f d r: 1.0, p 2 \_f d r: 0.04\right)$.

\subsection{Red de control ejecutivo}

Se observó aumento del ALFF en el núcleo caudado derecho en el grupo EA, con tamaño del efecto mediano de valor -0.43. Por otro lado, se obtuvo un incremento en la entropía del grupo EA en comparación con el grupo CN en las siguientes regiones: Crus I derecho con tamaño del efecto - 0.38 , giro frontal medio derecho con tamaño del efecto -0.35 , núcleo caudado derecho con tamaño del efecto -0.37 y en la región que involucra al Crus I, Crus II y lóbulo VI derecho con tamaño del efecto -0.38. Las diferencias descritas previamente presentaron significancia estadística sin corrección FDR (Tabla 4, Figura 3 y Figura 4).

No se observaron diferencias entre grupos para la métrica fALFF. El diagrama de cajas puede visualizarse en la Figura S3 del apéndice. 


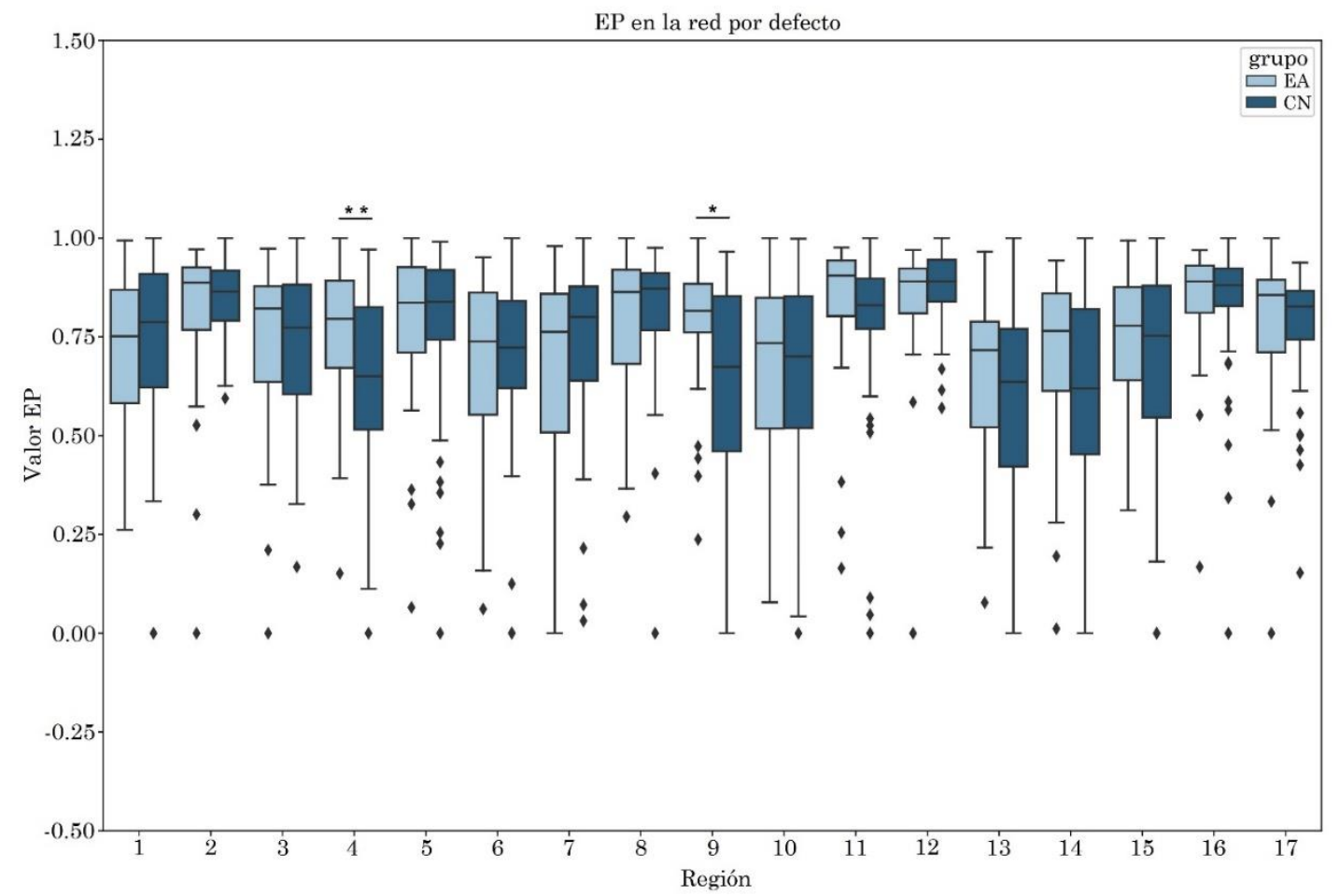

Figura 2. EP en la red por defecto, diferencia estadísticamente significativa ${ }^{*} \mathrm{p} \leq 0.05$ en la región que involucra a la corteza retroesplenial izquierda y la corteza del cíngulo posterior, ** sin corrección en la región que involucra la corteza cingulada posterior y precuña. Fuente: elaboración propia.

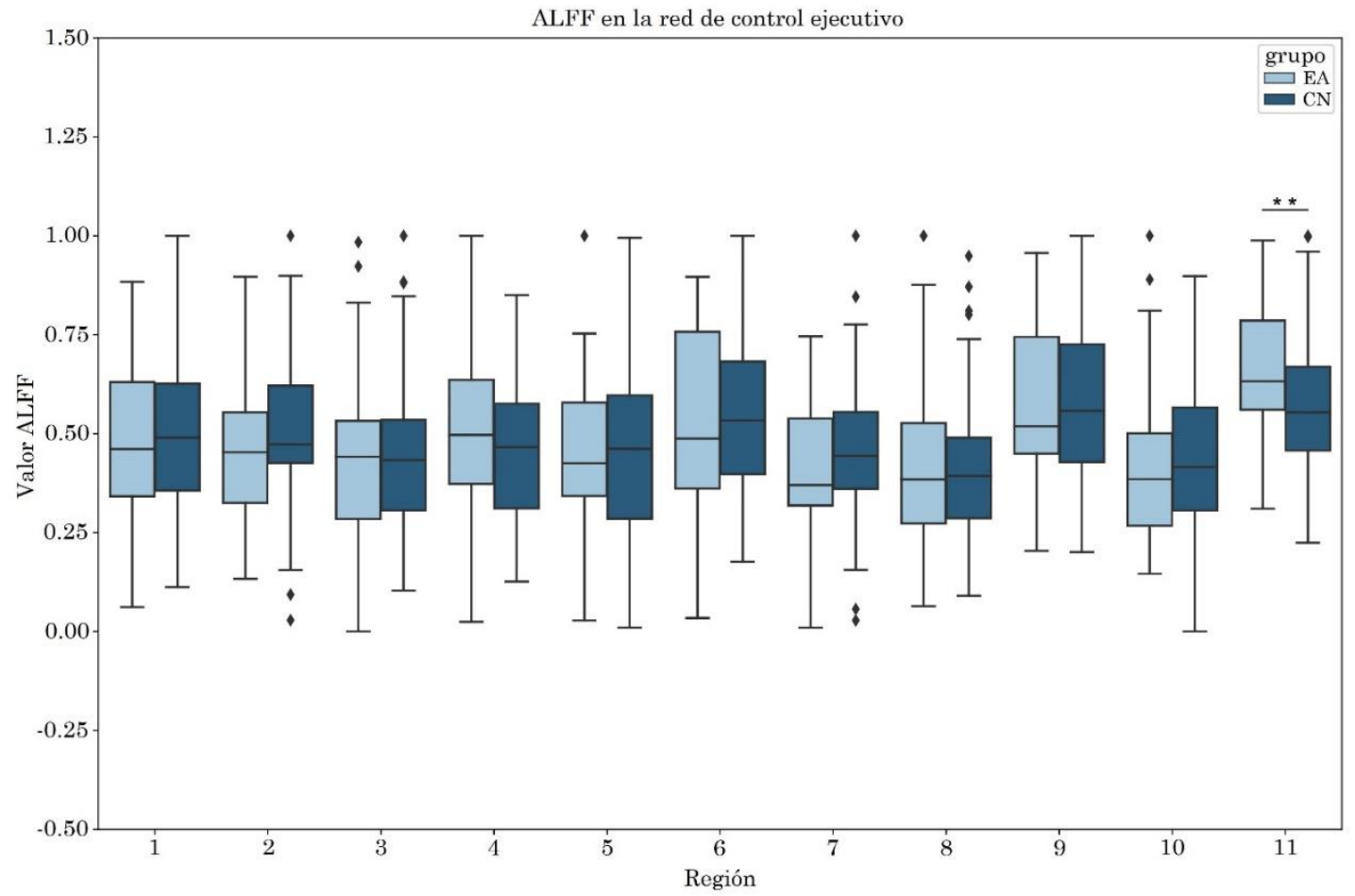

Figura 3. ALFF: en la red de control ejecutivo se encontraron diferencias estadísticamente significativas ** sin corrección en el núcleo caudado derecho. Fuente: elaboración propia. 
Tabla 4. Valores-p y tamaño del efecto para las 11 regiones de la red de control ejecutivo Fuente: elaboración propia.

\begin{tabular}{|c|c|c|c|c|c|c|c|c|c|}
\hline \multirow{2}{*}{ ROI } & \multicolumn{3}{|c|}{ ALFF } & \multicolumn{3}{|c|}{ fALFF } & \multicolumn{3}{|c|}{$\mathrm{PE}$} \\
\hline & p1_unc & p2_unc & ES & p1_unc & p2_unc & ES & p1_unc & p2_unc & ES \\
\hline 1 & .47 & .53 & .01 & .88 & .12 & -.24 & .34 & .66 & .09 \\
\hline 2 & .21 & .79 & .17 & .41 & .59 & .05 & .56 & .44 & -.04 \\
\hline 3 & .41 & .59 & .06 & .20 & .80 & .18 & .24 & .76 & .16 \\
\hline 4 & .85 & .15 & -.23 & .82 & .18 & -.20 & .78 & .22 & -.17 \\
\hline 5 & .71 & .29 & -.12 & .58 & .42 & -.04 & .97 & .03 & -.38 \\
\hline 6 & .41 & .59 & .05 & .83 & .17 & -.21 & .29 & .71 & .12 \\
\hline 7 & .12 & .88 & .25 & .08 & .92 & .31 & .95 & .05 & -.35 \\
\hline 8 & .52 & .48 & -.01 & .40 & .60 & .06 & .17 & .83 & .22 \\
\hline 9 & .39 & .61 & .06 & .14 & .86 & .25 & .73 & .27 & -.13 \\
\hline 10 & .41 & .59 & .05 & .79 & .21 & -.17 & .96 & .04 & -.38 \\
\hline 11 & .98 & .02 & -.43 & .13 & .87 & .25 & .96 & .04 & -.37 \\
\hline
\end{tabular}

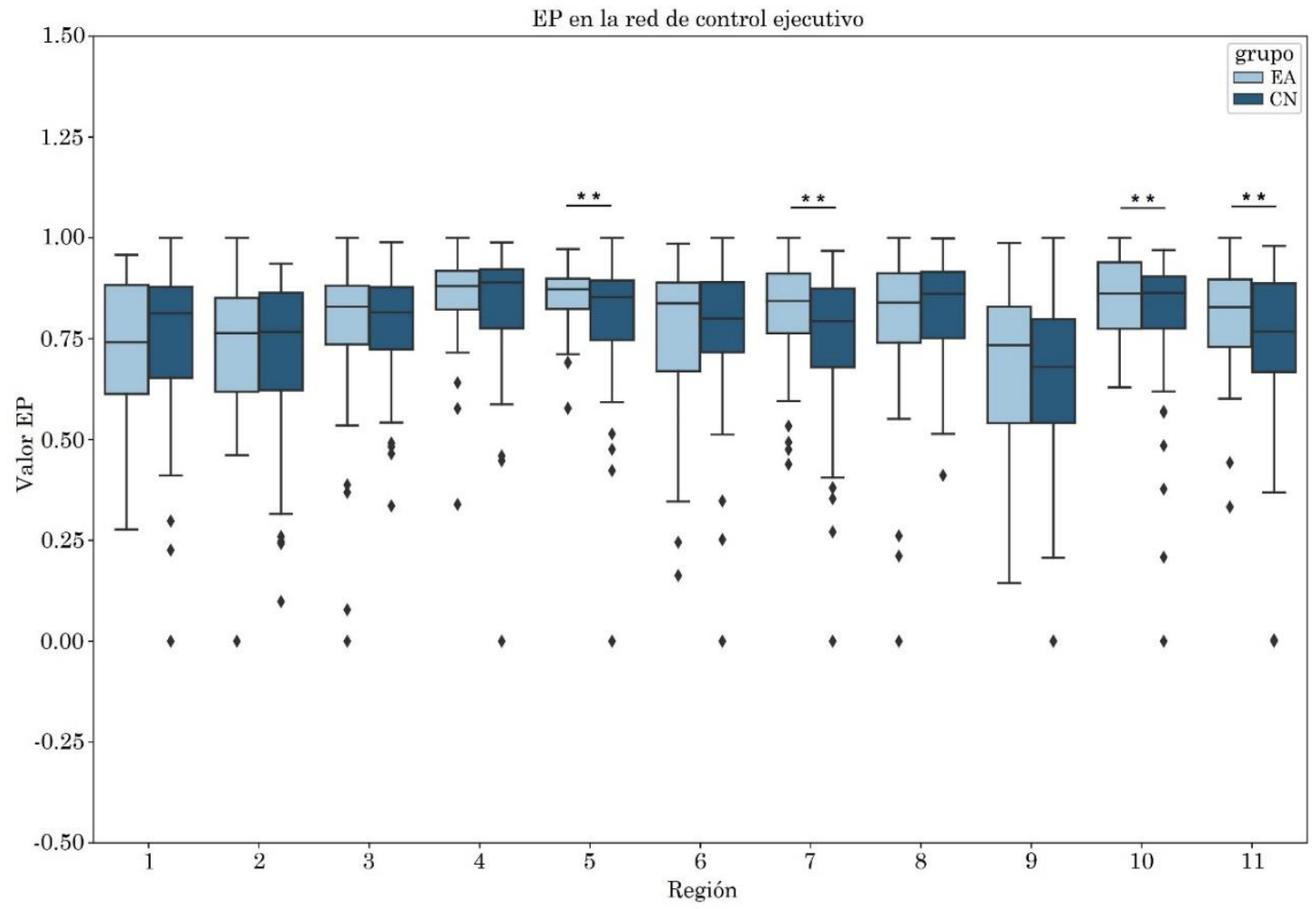

Figura 4 EP: en la red de control ejecutivo se encontraron diferencias estadísticamente significativas

** sin corrección en la región Crus I derecho, giro frontal medio derecho, la región que involucra al Crus I, Crus II y lóbulo VI derecho, y en el núcleo caudado derecho. Fuente: elaboración propia. 
Los valores obtenidos al correlacionar las métricas con las escalas clínicas CDR-SB y MMSE para las regiones de la red de control ejecutivo se encuentran en las Tablas S1 y S2, respectivamente del apéndice.

\section{DISCUSIÓN}

En este estudio se investigó el potencial de la métrica entropía por permutaciones para extraer información sobre la complejidad cerebral en regiones que conforman la red por defecto y la red de control ejecutivo, se comparó su desempeño con las métricas espectrales ALFF y fALFF en pacientes con EA y sujetos sanos. Se encontró un aumento de la EP en pacientes con EA en ambas redes, adicionalmente se encontró un aumento en el ALFF en pacientes con EA en la red de control ejecutivo.

$\mathrm{Al}$ analizar la red por defecto en pacientes, se halló un incremento de la EP en la región que involucra a la corteza cingulada posterior y la precuña. Por otro lado, el estudio sobre la red de control ejecutivo reveló un incremento de la ALFF en el núcleo caudado y un incremento de la EP en las regiones del hemisferio derecho: giro frontal medio, núcleo caudado y la región que comprende al Crus I, Crus II y el lóbulo VI. En línea con estos resultados se ha reportado que la desorganización en la conectividad funcional de esta región es de importancia para el desarrollo de demencia en la EA [31]. La corteza posterior del cíngulo es considerada una región clave de la red por defecto en la que se han reportado cambios en la conectividad cerebral en EA [32], con alto poder discriminatorio entre esta y sujetos controles [33]. Desde un punto de vista patológico, la precuña y la corteza cingulada posterior se han asociado con el desarrollo completo de la EA, en donde se ha reportado retención de proteína tau y astrogliosis, además de alteraciones significativas en la conectividad funcional [31]. Un estudio estructural de resonancia magnética reportó que la corteza posterior del cíngulo es una de las regiones más vulnerables involucrada en la patofisiología de la EA. Se identificó un deterioro acelerado de múltiples dominios cognitivos en individuos con una mayor tasa de atrofia del volumen de la sustancia gris de esta región [34]. En un metaanálisis de conectividad con rs-fMRI se ha reportado en sujetos con EA sin conectividad entre la red por defecto y el sistema límbico, dado principalmente por las áreas del precuneus y el cíngulo posterior [8]. Otro estudio que evaluaba la conectividad intrared por defecto mostró aumento de la conectividad entre el cíngulo posterior con la corteza parahipocampal y la precuña en personas con EA comparada con controles [35]. Sin embargo, se debe tener en cuenta que los hallazgos de conectividad en reposo en EA son contradictorios. $\mathrm{Al}$ parecer, la conectividad en la red por defecto se puede modificar según el estadio de la demencia, pudiendo haber una disminución en áreas posteriores parietales y temporales al inicio de los síntomas y un aumento en áreas anteriores frontales con el progreso de la enfermedad, no se ha esclarecido si es debido a una posible compensación o un cambio debido a la fisiopatología misma del depósito de proteínas anormales [36].

Contrario a los resultados de esta investigación, estudios previos de rs-fMRI [19], [37], [38] han reportado reducción de la complejidad en EA en comparación con sujetos sanos. Uno de esos estudios [19] halló disminución de la EP en el grupo EA al comparar con el grupo control en regiones de los lóbulos temporal, occipital y frontal (hemisferio derecho: giro temporal inferior, cuña, giro occipital medio y giro occipital superior; hemisferio izquierdo: giro frontal medio, giro frontal superior, giro cingulado anterior y cuña). Por otro lado, el mismo estudio reportó incremento de la EP en el grupo de deterioro cognitivo leve de etapa temprana al comparar con el grupo control en las regiones del hemisferio derecho: giro temporal inferior, giro frontal medio, cuña, giro occipital medio y giro occipital superior. Es 
importante resaltar que el estudio referenciado no partió del estudio de regiones que forman parte de las redes cerebrales en reposo que se ven mayormente afectadas en la enfermedad de Alzheimer; en su lugar, el análisis fue realizado sobre 8 regiones pertenecientes a 5 clústeres que mostraron diferencias significativas luego de obtener la métrica EP sobre todos los voxeles del cerebro. Los datos del estudio fueron tomados de la iniciativa ADNI. Por otro lado, el estudio de [37] utilizó la métrica entropía multiescala para cuantificar la complejidad de las señales BOLD en sujetos con deterioro cognitivo leve temprano, deterioro cognitivo leve tardío y sujetos controles tomados de la iniciativa ADNI. El estudio reportó disminución de la complejidad en la región del giro fusiforme izquierdo y en la corteza cingulada anterior rostral en el grupo de deterioro cognitivo leve temprano. El enfoque metodológico consistió en la generación de mapas de entropía multiescala para cada sujeto sobre todos los voxeles del cerebro. Con respecto al estudio de [38], estos utilizaron la métrica entropía multiescala para cuantificar la complejidad de las señales BOLD en sujetos con deterioro cognitivo leve y sujetos controles reclutados en la Clínica de Memoria del Departamento de Geriatría del Hospital Universitario Karolinska, en Huddinge, Suecia. El análisis se realizó sobre regiones de la red por defecto definida por [25], de las cuales reportó disminución de la entropía a nivel global y a nivel nodal reportó reducción en el hipocampo derecho. Los resultados se reportan sin corrección para comparaciones múltiples. Como limitaciones comunes de los estudios citados previamente se resalta que la iniciativa ADNI no proporcionó públicamente información sobre los factores de riesgo relacionados con la EA [19] y se indica que estudios previos han sugerido que el criterio diagnóstico de deterioro cognitivo leve de la iniciativa ADNI tiene alta tasa de falsos positivos [37].

El estudio de [39] evaluó el impacto del envejecimiento en la demencia desde un enfoque celular, en el que la EA de aparición tardía puede verse como una manifestación de producción de energía reducida, dando lugar a un aumento en la entropía. Los autores sugieren que la acumulación de modificaciones relacionadas con la edad, así como el agotamiento de las mitocondrias, podrían ser responsables de la escasa capacidad del cerebro para adaptar sus estructuras y funciones cerebrales. Por otro lado, la relación entre la entropía durante el envejecimiento saludable y la EA ha sido explorada recientemente por medio de rs-fMRI y la métrica de mapeo de entropía cerebral (BEN) [40] en grupos de individuos controles ( $\mathrm{n}=54$, edad: $65-95$ años), controles con compromiso de la memoria $(\mathrm{n}=27$, edad 65-95 años), deterioro cognitivo leve temprano ( $n=58$, edad: 56-89 años), deterioro cognitivo leve tardío ( $\mathrm{n}=38,57-88$ años) y sujetos con EA ( $\mathrm{n}=34,56-87$ años). El estudio referenciado reportó el siguiente comportamiento de la $\mathrm{BEN}$ en los cuatro grupos de interés. La comparación se describe con respecto al grupo control: leve aumento en los individuos controles con compromiso de la memoria, incremento más notorio en el grupo de deterioro cognitivo leve temprano. Para la etapa de deterioro cognitivo leve tardío se reportó una disminución leve que posteriormente se convirtió en una reducción más notoria en el grupo de EA, la reducción en la BEN en las etapas tardías de la demencia son relacionadas por el autor con un fenómeno fallido de compensación [40]. Según [41] y [42], la reducción notoria de la BEN presente en las etapas tardías de la demencia da lugar al deterioro acelerado de funciones cerebrales que requieren niveles de entropía altos para ser flexibles funcionalmente. Los hallazgos de este estudio, descritos como demencia tipo Alzheimer en la sección 2.1, están en línea con el comportamiento descrito por el grupo de deterioro cognitivo leve de inicio temprano en el estudio realizado por [40], resalta este aspecto por ser el único estudio que describe el comportamiento de la entropía en 5 estadios cognitivos de interés en el estudio de la EA. Sin embargo, a través de la referencia [38] se evidencian contradicciones en los hallazgos de estudios realizados a partir de datos de rs-fMRI en EA con el objetivo de cuantificar la complejidad de las señales BOLD en EA, lo que sugiere que es necesario 
realizar estudios adicionales en el campo, que además incluyan seguimiento longitudinal y proporcionen información adicional sobre los criterios de selección de sujetos.

La ALFF es una métrica que permite identificar la actividad neuronal espontánea de regiones específicas del cerebro a través de la intensidad de la señal BOLD. Esta ha sido utilizada como métrica característica para mejorar el desempeño de algoritmos de aprendizaje de máquina para la discriminación de sujetos con DCL y EA de individuos cognitivamente normales [13]. La ALFF y las métricas de entropía no están relacionadas teóricamente, sin embargo, en este estudio se encontró un aumento en la ALFF y la PE en el núcleo caudado. El estudio de [43] realizado con una cohorte de sujetos sanos reportó correlación positiva de magnitud mediana a alta entre el BEN y la fALFF en la corteza orbitofrontal y la corteza temporal inferior posterior. Por otro lado, reportó correlaciones negativas de gran magnitud entre el BEN y la fALFF en la corteza visual, corteza temporal inferior anterior, red motora, precuña y la corteza parietal lateral, lo que sugiere que la fALFF y la BEN son mutuamente independientes. El estudio referenciado previamente sugiere que la BEN puede proveer información más completa de la función cerebral, especialmente en regiones donde no hay asociaciones entre la BEN y la fALFF, en línea con los hallazgos de nuestro estudio.

Dentro de las limitaciones de esta investigación, se encuentra el uso de grupos no pareados por sexo y edad. Es importante destacar que OASIS-3 es una base de datos que incluye sujetos con edades comprendidas entre los 42 años y los 95 años que fueron reclutados en el transcurso de 30 años. Algunos de los sujetos incluidos en este estudio fueron inicialmente etiquetados como cognitivamente normales que desarrollaron demencia por EA con el tiempo y otros ingresaron a la iniciativa OASIS-3 con diagnóstico de demencia tipo Alzheimer, para ambos casos la revisión realizada por los expertos clínicos permitió excluir sujetos con etiqueta EA que podían tener síntomas explicados por otras condiciones clínicas o no contar con un diagnóstico claro, a partir de la información proporcionada por la historia clínica disponible en la base de datos. Se debe tener en cuenta que en la base de datos no se especifica el estadio clínico de la demencia en la que se encuentran los sujetos, por lo que se tuvo que determinar a partir de mediciones de escalas que, si bien son fiables, no igualan el criterio clínico. Los enfoques de análisis reportados por estudios previos limitan la comparación de los hallazgos de este estudio, principalmente por la incertidumbre acerca del estadio de los sujetos clasificados como EA o deterioro cognitivo leve y tardío de la iniciativa ADNI, además del uso de métricas diferentes a la EP para cuantificar la complejidad de las señales BOLD. Además, se encontraron algunas limitaciones relacionadas con la presencia de artefactos en las imágenes y cambios en el diagnóstico durante el seguimiento longitudinal que disminuyó la muestra inicial. Aunque el tamaño de las señales es de mayor duración en comparación al estudio de PE realizado por [19], la realización de este análisis sobre señales con más de 164 volúmenes sería beneficioso para evaluar la entropía en el continuum de la EA debido a los 24 estados definidos por la dimensión embebida igual a 4 .

Si bien las pruebas estadísticas dan una inferencia considerable de la relación entre dos o más variables, permitiendo una interpretabilidad dentro de los datos, su precisión predictiva no es su principal fortaleza, por lo que en investigaciones futuras se deberían considerar los efectos potenciales del aprendizaje de máquina para complementar los resultados presentados, donde se tendría la capacidad de generar un modelo a partir de la métrica no lineal PE y obtener predicciones reproducibles, con un análisis más robusto en la búsqueda de patrones discriminantes entre grupos, en este caso EA vs CN, que permitiría, además, obtener un mayor poder predictivo con la adición de mayor cantidad de muestras de las diferentes poblaciones y reducir la presencia de artefactos [44]. En consecuencia, de acuerdo a ciertos estudios, [16], [45], se ha sugerido el uso de métricas de conectividad 
funcional de fluctuaciones dinámicas no lineales como datos de base para el análisis, ya que proporciona una mejor caracterización patofisiológica de las redes neuronales en poblaciones con enfermedades neurodegenerativas, además de ser datos que pueden ser eficientemente manipulados por las redes neuronales artificiales, puesto que permiten el modelamiento de relaciones no lineales en campos de gran complejidad.

\section{CONCLUSIONES}

En el estudio actual se identificó incremento de la entropía en regiones de la red por defecto y la red de control ejecutivo en pacientes con EA. El hallazgo diferencial de mayor magnitud se manifestó en la corteza cingulada posterior y la precuña. La metodología implementada permitió demostrar la capacidad de la métrica entropía por permutaciones para capturar información sobre la función cerebral en la enfermedad de Alzheimer en comparación a las métricas espectrales. La métrica debe ser explorada en estudios longitudinales y en señales de mayor duración para evaluar su potencial para capturar patrones funcionales relacionados con la EA.

\section{AGRADECIMIENTOS}

Este trabajo fue apoyado por el Departamento Administrativo de Ciencias, Tecnología e Innovación (Colciencias), por el proyecto 'Identificación de Biomarcadores Preclínicos en Enfermedad de Alzheimer a través de un Seguimiento Longitudinal de la Actividad Eléctrica Cerebral en Poblaciones con Riesgo Genético', código 111577757635.

Los datos fueron proporcionados por OASIS-3: Principal Investigators: T. Benzinger, D. Marcus, J. Morris; NIH P50 AG00561, P30 NS09857781, P01 AG026276, P01 AG003991, R01 AG043434, UL1 TR000448, R01 EB009352.

Parte de la financiación de los autores fue aportada por el fortalecimiento de programas de ciencias médicas y de la salud con talento joven e impacto regional del Ministerio de Ciencia, Tecnología e Innovación (MinCiencias) - RC 752 - 2018. Estos recursos fueron administrados por la Corporación Académica para el Estudio de Patologías Tropicales de la Universidad de Antioquia.

\section{CONFLICTOS DE INTERÉS}

El proyecto no presenta ningún conflicto de interés.

\section{CONTRIBUCIÓN DE LOS AUTORES}

Aura C. Puche: diseño del experimento, procesamiento de datos, análisis de resultados y preparación inicial del manuscrito. Revisión, edición y aprobación del documento final.

John Fredy Ochoa Gómez: diseño del experimento y análisis de resultados; Revisión, edición y aprobación del documento final.

Yésika Alexandra Agudelo L.: análisis de resultados y preparación inicial del manuscrito. Revisión, edición y aprobación del documento final. 
Jan Karlo Rodas Marín (experto clínico): selección de sujetos y análisis de resultados. Revisión, edición y aprobación del documento final.

Carlos Tobón Quintero (experto clínico): selección de sujetos y análisis de resultados. Revisión, edición y aprobación del documento final.

\section{REFERENCIAS}

[1] World Health Organization, "The Global Dementia Observatory Reference Guide World Health Organization," Geneva, Switzerland. 2018. URL

[2] Y. Takeuchi; Y. Ariza-Araujo; S. Prada, "P3-349: Prevalence estimates of dementia in colombia (2005-2020): transitions and stage of disease," Alzheimer's Dement., vol. 10, no. 4S, pp. 758-758, Jul. 2014. https://doi.org/10.1016/j.jalz.2014.05.1442

[3] S. I. Prada; Y. Takeuchi; Y. Ariza, "Costo monetario del tratamiento de la enfermedad deAlzheimer en Colombia”, Acta Neurol Colomb., vol. 30, no. 4, Oct. 2014. URL

[4] M. A. Busche; B. T. Hyman, "Synergy between amyloid-B and tau in Alzheimer's disease," Nature Neuroscience, vol. 23, no. 10, pp. 1183-1193, Oct.2020. https://doi.org/10.1038/s41593-020-0687-6

[5] L. Vermunt et al., "Duration of preclinical, prodromal, and dementia stages of Alzheimer's disease in relation to age, sex, and APOE genotype," Alzheimer's Dement., vol. 15, no. 7, pp. 888-898, Jun. 2019. https://doi.org/10.1016/j.jalz.2019.04.001

[6] B. C. Dickerson; F. Agosta; M. Filippi, "fMRI in Neurodegenerative Diseases: From Scientific Insights to Clinical Applications," in fMRI techniques and protocols, Humana Press, New York, NY, 2016, pp. 699739. https://doi.org/10.1007/978-1-4939-5611-1_23

[7] F. Agosta; M. Pievani; C. Geroldi; M. Copetti; G. B. Frisoni; M. Filippi, "Resting state fMRI in Alzheimer's disease: beyond the default mode network," Neurobiol. Aging, vol. 33, no. 8, pp. 1564-1578, Aug. 2012. https://doi.org/10.1016/j.neurobiolaging.2011.06.007

[8] A. P. Badhwar; A. Tam; C. Dansereau; P. Orban; F. Hoffstaedter; P. Bellec, "Resting-state network dysfunction in Alzheimer's disease: A systematic review and meta-analysis," Alzheimer's Dement. Diagnosis, Assess. Dis. Monit., vol. 8, no. 1, pp. 73-85, Apr. 2017. https://doi.org/10.1016/j.dadm.2017.03.007

[9] M. Weiler et al., "Default mode, executive function, and language functional connectivity networks are compromised in mild Alzheimer's disease," Curr. Alzheimer Res., vol. 11, no. 3, pp. 274-282, Mar. 2014. https://doi.org/10.2174/1567205011666140131114716

[10] Q. Zhao; H. Lu; H. Metmer; W. X. Y. Li; J. Lu, "Evaluating functional connectivity of executive control network and frontoparietal network in Alzheimer's disease,” Brain Res., vol. 1678, pp. 262-272, Jan. 2018. https://doi.org/10.1016/j.brainres.2017.10.025

[11] E. L. Dennis; P. M. Thompson, "Functional Brain Connectivity Using fMRI in Aging and Alzheimer's Disease,” Neuropsychol. Rev., vol. 24, no. 1, pp. 49-62, Mar. 2014. https://doi.org/10.1007/s11065-014-9249$\underline{6}$

[12] H. Lv et al., "Resting-State Functional MRI: Everything That Nonexperts Have Always Wanted to Know," Am. J. Neuroradiol., vol 39. no. 8, pp. 1390-1399, Aug. 2018. https://doi.org/10.3174/ajnr.A5527

[13] L. Yang et al., "Gradual Disturbances of the Amplitude of Low-Frequency Fluctuations (ALFF) and Fractional ALFF in Alzheimer Spectrum," Front. Neurosci., vol. 12, Dec. 2018. https://doi.org/10.3389/fnins.2018.00975

[14] R. A. Poldrack, "The role of fMRI in Cognitive Neuroscience: where do we stand?," Curr. Opin. Neurobio., vol. 18, no. 2, pp. 223-227, Apr. 2008. https://doi.org/10.1016/j.conb.2008.07.006

[15] N. M. Timme; C. Lapish, "A Tutorial for Information Theory in Neuroscience,” eNeuro, vol. 5, no. 3, May 2018. https://doi.org/10.1523/ENEURO.0052-18.2018

[16] S. Moguilner et al., "Dynamic brain fluctuations outperform connectivity measures and mirror pathophysiological profiles across dementia subtypes: A multicenter study," Neuroimage, vol. 225, p. 117522, Jan. 2021. https://doi.org/10.1016/j.neuroimage.2020.117522

[17] A. C. Yang; S.-J. Tsai; C.-P. Lin; C.-K. Peng, "A Strategy to Reduce Bias of Entropy Estimates in RestingState fMRI Signals," Front. Neurosci., vol. 12, p. 398, Jun. 2018. https://doi.org/10.3389/fnins.2018.00398

[18] C. Bandt; B. Pompe, "Permutation Entropy: A Natural Complexity Measure for Time Series," Phys. Rev. Lett., vol. 88, no. 17, Dec. 2001. URL

[19] B. Wang et al., "Decreased Complexity in Alzheimer's Disease: Resting-State fMRI Evidence of Brain Entropy Mapping," Front. Aging Neurosci., vol. 9, Nov. 2017. https://doi.org/10.3389/fnagi.2017.00378

[20] J. Sun et al., "Complexity analysis of EEG, MEG, and fMRI in mild cognitive impairment and Alzheimer's disease: A review," Entropy, vol. 22, no. 2, Feb. 2020. https://doi.org/10.3390/e22020239 
[21] P. J. LaMontagne et al., "OASIS-3: Longitudinal Neuroimaging, Clinical, and Cognitive Dataset for Normal Aging and Alzheimer Disease," medRxiv, pp. 2-37, Dec. 2019. https://doi.org/10.1101/2019.12.13.19014902

[22] S. E. O'Bryant et al., "Staging dementia using clinical dementia rating scale sum of boxes scores: A Texas Alzheimer's research consortium study," Arch. Neurol., vol. 65, no. 8, pp. 1091-1095, Aug. 2008. https://doi.org/10.1001/archneur.65.8.1091

[23] OASIS, "OASIS-3: Imaging Methods \& Data Dictionary,” Apr. 2018. URL

[24] S. Whitfield-Gabrieli; A. Nieto-Castanon, "Conn: A Functional Connectivity Toolbox for Correlated and Anticorrelated Brain Networks,” Brain Connect., vol. 2, no. 3, pp. 125-141, Jun. 2012. https://doi.org/10.1089/brain.2012.0073

[25] W. R. Shirer; S. Ryali; E. Rykhlevskaia; V. Menon; M. D. Greicius, "Decoding Subject-Driven Cognitive States with Whole-Brain Connectivity Patterns," Cereb. Cortex, vol. 22, no. 1, pp. 158-165, May. 2011. https://doi.org/10.1093/cercor/bhr099

[26] C.-G. Yan; X.-D. Wang; X.-N. Zuo; Y.-F. Zang, "DPABI: Data Processing \& Analysis for (Resting-State) Brain Imaging," Neuroinform., vol. 14, no. 3, pp. 339-351, Jul. 2016. https://doi.org/10.1007/s12021-0169299-4

[27] X.-N. Zuo et al., "The oscillating brain: complex and reliable.," Neuroimage, vol. 49, no. 2, pp. 1432-1445, Jan. 2010. https://doi.org/10.1016/j.neuroimage.2009.09.037

[28] M. Riedl; A. Müller; N. Wessel, "Practical considerations of permutation entropy: A tutorial review," Eur. Phys. J. Spec. Top., vol. 222, no. 2, pp. 249-262, Jun. 2013. https://doi.org/10.1140/epjst/e2013-01862-7

[29] E. Glerean et al., "Reorganization of functionally connected brain subnetworks in high-functioning autism," Hum. Brain Mapp., vol. 37, pp. 1066-1079, Dec. 2015. https://doi.org/10.1002/hbm.23084

[30] H. Hentschke; M. C. Stüttgen, "Computation of measures of effect size for neuroscience data sets.," Eur. J. Neurosci., vol. 34, no. 12, pp. 1887-1894, Dec. 2011. https://doi.org/10.1111/j.1460-9568.2011.07902.x

[31] T. Yokoi et al., "Involvement of the precuneus/posterior cingulate cortex is significant for the development of Alzheimer's disease: A PET (THK5351, PiB) and resting fMRI study," Front. Aging Neurosci., vol. 10, Oct. 2018. https://doi.org/10.3389/fnagi.2018.00304

[32] K. N. H. Dillen et al., "Aberrant functional connectivity differentiates retrosplenial cortex from posterior cingulate cortex in prodromal Alzheimer's disease,” Neurobiol. Aging, vol. 44, pp. 114-126, Aug. 2016. https://doi.org/10.1016/j.neurobiolaging.2016.04.010

[33] W. Koch et al., "Diagnostic power of default mode network resting state fMRI in the detection of Alzheimer's disease," Neurobiol. Aging, vol. 33, no. 3, pp. 466-478, Mar. 2012.

https://doi.org/10.1016/j.neurobiolaging.2010.04.013

[34] P. L. Lee et al., "Posterior Cingulate Cortex Network Predicts Alzheimer's Disease Progression," Front. Aging Neurosci., vol. 12, Dec. 2020. https://doi.org/10.3389/fnagi.2020.608667

[35] L. Chenxi et al., "Abnormal Brain Network Connectivity in a Triple-Network Model of Alzheimer's Disease,” J. Alzheimers. Dis., vol. 69, no. 1, pp. 237-252, 2019. https://doi.org/10.3233/JAD-181097

[36] W. Jagust, "Imaging the evolution and pathophysiology of Alzheimer disease," Nat. Rev. Neurosci., vol. 19, no. 11, pp. 687-700, Nov. 2018. https://doi.org/10.1038/s41583-018-0067-3

[37] H. Zheng; K. Onoda; A. Nagai; S. Yamaguchi, "Reduced Dynamic Complexity of BOLD Signals Differentiates Mild Cognitive Impairment From Normal Aging," Front. Aging Neurosci., vol. 12, Apr. 2020. https://doi.org/10.3389/fnagi.2020.00090

[38] M. Grieder; D. J. J. Wang; T. Dierks; L.-O. Wahlund; K. Jann, "Default Mode Network Complexity and Cognitive Decline in Mild Alzheimer's Disease," Front. Neurosci., vol. 12, Oct. 2018. https://doi.org/10.3389/fnins.2018.00770

[39] V. Boccardi; C. Comanducci; M. Baroni; P. Mecocci, "Of energy and entropy: The ineluctable impact of aging in old age dementia," Int. J. Mol. Sci., vol. 18, no. 12, Dec. 2017. https://doi.org/10.3390/ijms18122672

[40] Z. Wang, "Brain Entropy Mapping in Healthy Aging and Alzheimer's Disease,” Front. Aging Neurosci., vol. 12, p. 372, Nov. 2020. https://doi.org/10.3389/fnagi.2020.596122

[41] E. Tagliazucchi; P. Balenzuela; D. Fraiman; D. R. Chialvo, "Criticality in large-scale brain fMRI dynamics unveiled by a novel point process analysis," Front. Physiol., vol. 3, Feb. 2012. https://doi.org/10.3389/fphys.2012.00015

[42] A. Haimovici; E. Tagliazucchi; P. Balenzuela; D. R. Chialvo, "Brain Organization into Resting State Networks Emerges at Criticality on a Model of the Human Connectome.”, Phys. Rev. Lett., vol. 110, no.17, Apr. 2013. https://doi.org/10.1103/PhysRevLett.110.178101

[43] D. Song; D. Chang, J. Zhang; Q. Ge; Y. F. Zang; Z. Wang, "Associations of brain entropy (BEN) to cerebral blood flow and fractional amplitude of low-frequency fluctuations in the resting brain," Brain Imaging Behav., vol. 13, no. 5, pp. 1486-1495, Oct. 2019. https://doi.org/10.1007/s11682-018-9963-4

[44] L. Mera-Jiménez; J. F. Ochoa-Gómez, "Redes neuronales convolucionales para la clasificación de componentes independientes de rs-fMRI," TecnoLógicas, vol. 24, no. 50, pp. 97-115, Jan. 2021.

https://doi.org/10.22430/22565337.1626 
[45] R. Liégeois et al., "Resting brain dynamics at different timescales capture distinct aspects of human behavior," Nat. Commun., vol. 10, no. 1, May. 2019. https://doi.org/10.1038/s41467-019-10317-7 


\section{APÉNDICE}

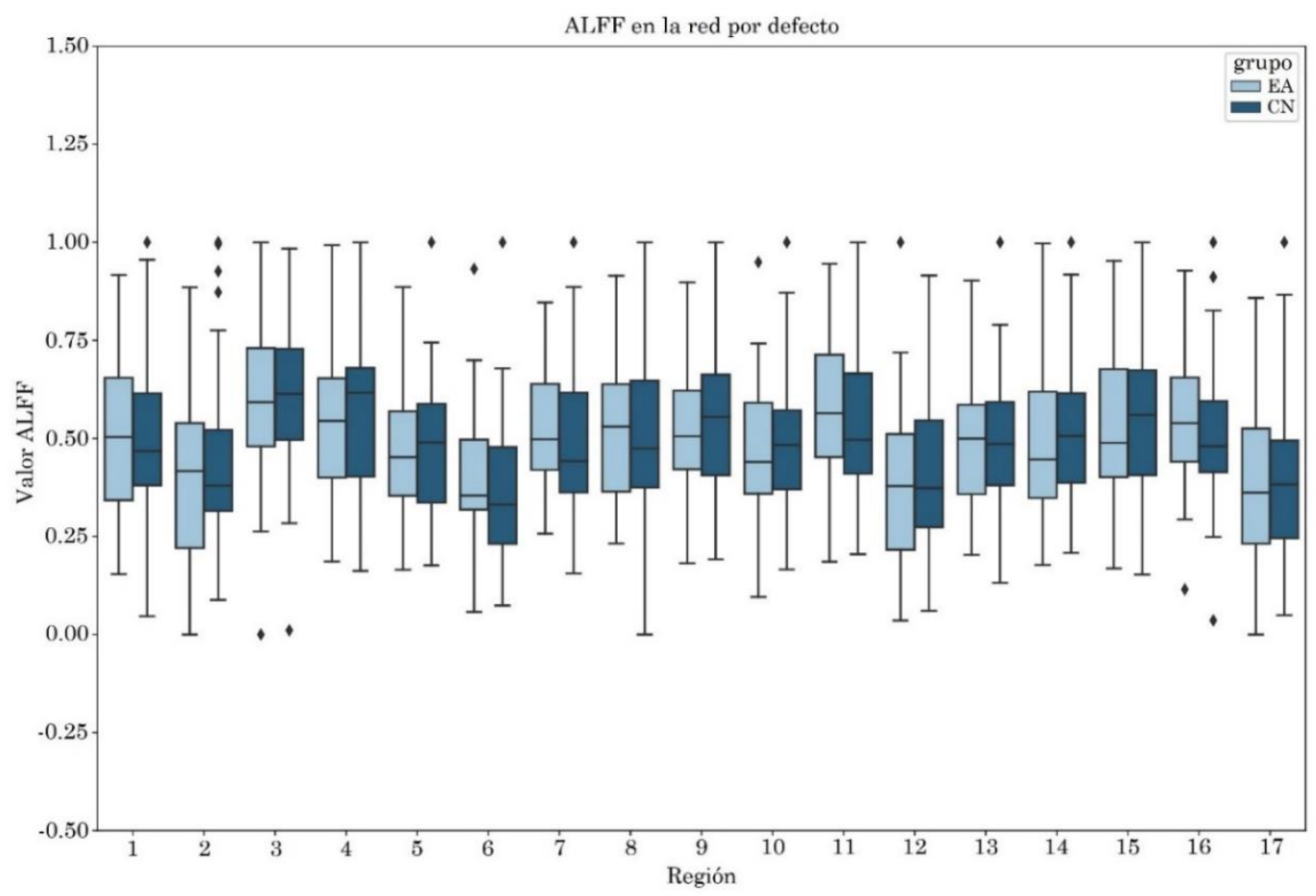

Figura S1. ALFF en la red por defecto, sin hallazgos estadísticamente significativos.

Fuente: elaboración propia.

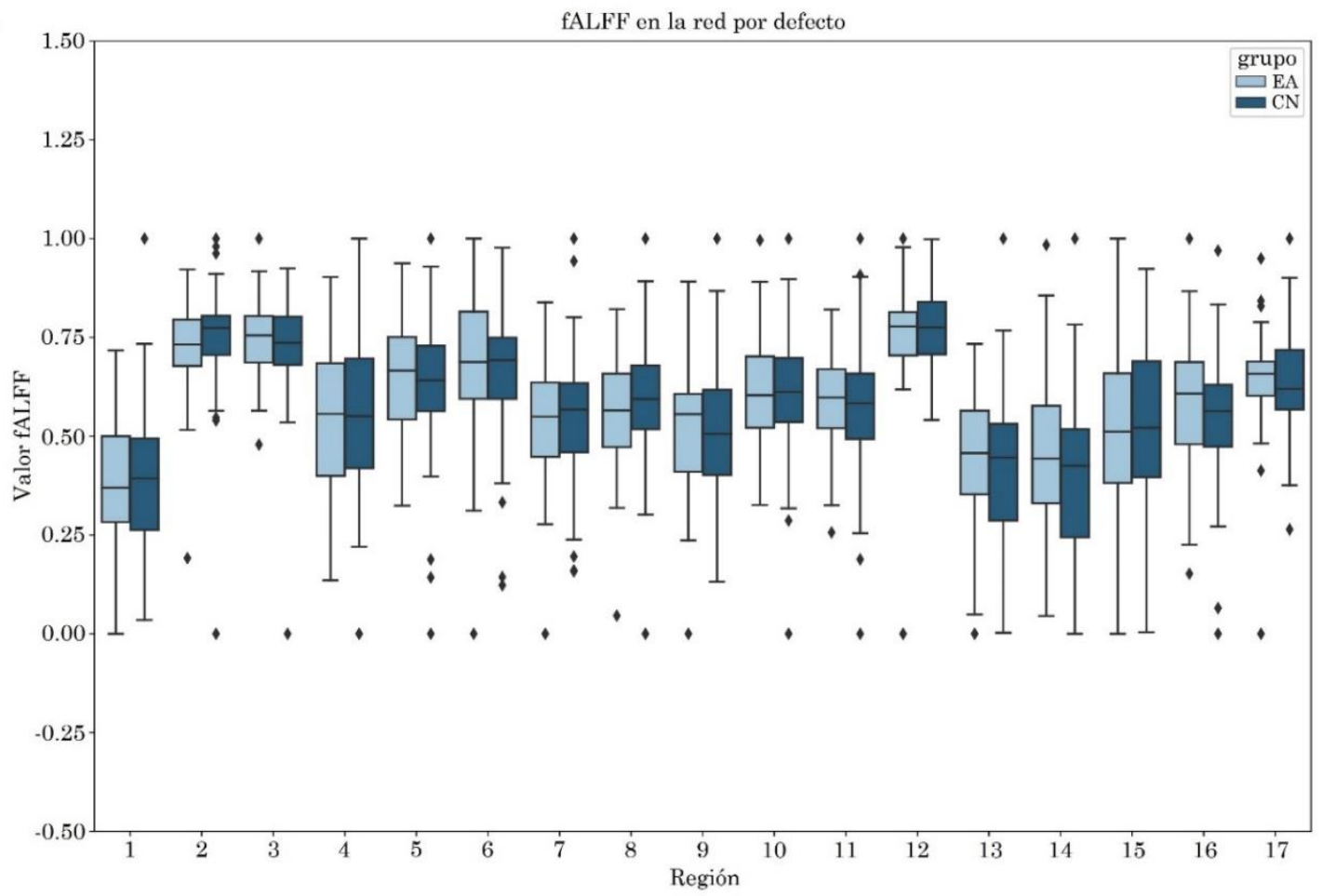

Figura S2. fALFF en la red por defecto, sin hallazgos estadísticamente significativos.

Fuente: elaboración propia. 


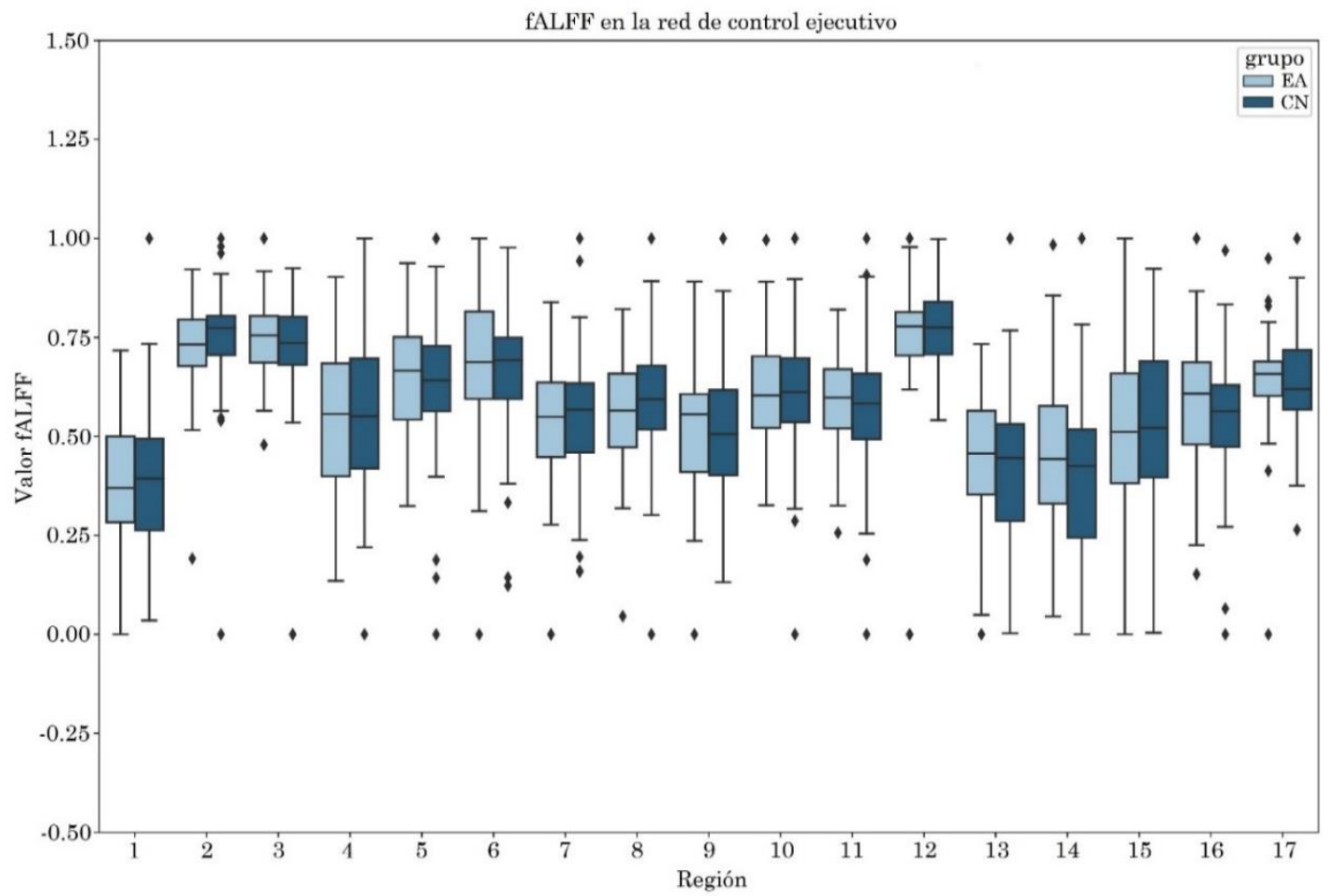

Figura S3. fALFF en la red de control ejecutivo, sin hallazgos estadísticamente significativos.

Fuente: elaboración propia.

Tabla S1. Correlación de las métricas con la escala CDR-SB para las regiones de la red por defecto. Fuente: elaboración propia.

\begin{tabular}{|c|c|c|c|c|c|c|}
\hline \multirow{2}{*}{ ROI } & \multicolumn{2}{|c|}{ ALFF } & \multicolumn{2}{|c|}{ Falff } & \multicolumn{2}{|l|}{$\mathrm{PE}$} \\
\hline & $r$ & $\mathrm{P}$ & $\mathrm{r}$ & $\mathrm{P}$ & $\mathrm{r}$ & $\mathrm{p}$ \\
\hline 1 & .01 & .94 & -.13 & .45 & -.14 & .41 \\
\hline 2 & -.10 & .55 & -.27 & .11 & -.04 & .83 \\
\hline 3 & .07 & .66 & -.12 & .48 & .04 & .82 \\
\hline 4 & .06 & .74 & .05 & .79 & .16 & .34 \\
\hline 5 & .10 & .58 & .13 & .46 & -.17 & .31 \\
\hline 6 & .05 & .76 & -.05 & .79 & .02 & .92 \\
\hline 7 & .05 & .77 & .02 & .90 & -.13 & .45 \\
\hline 8 & .06 & .72 & -.01 & .96 & .24 & .17 \\
\hline 9 & .07 & .70 & -.01 & .95 & .06 & .75 \\
\hline 10 & .13 & .44 & -.01 & .94 & .01 & .97 \\
\hline 11 & .09 & .59 & .25 & .15 & -.16 & .34 \\
\hline 12 & -.06 & .73 & .01 & .94 & .12 & .49 \\
\hline 13 & .11 & .54 & .08 & .66 & .03 & .86 \\
\hline 14 & .10 & .55 & .03 & .84 & .15 & .38 \\
\hline 15 & .04 & .80 & .06 & .71 & -.06 & .73 \\
\hline 16 & .05 & .77 & -.03 & .87 & -.20 & .24 \\
\hline 17 & .05 & .76 & .27 & .12 & .21 & .21 \\
\hline
\end{tabular}


Tabla S2. Correlación de las métricas con la escala MMSE para las regiones de la red por defecto.

Fuente: elaboración propia.

\begin{tabular}{|c|c|c|c|c|c|c|}
\hline \multirow{2}{*}{ ROI } & \multicolumn{2}{|c|}{$\mathrm{ALFF}$} & \multicolumn{2}{|c|}{ fALFF } & \multicolumn{2}{|l|}{$\mathrm{PE}$} \\
\hline & $r$ & $\mathrm{P}$ & $\mathrm{r}$ & $\mathrm{P}$ & $\mathrm{r}$ & $\mathrm{p}$ \\
\hline 1 & .11 & .53 & .12 & .48 & .07 & .68 \\
\hline 2 & .07 & .68 & .03 & .88 & .12 & .47 \\
\hline 3 & .17 & .32 & -.06 & .74 & .07 & .67 \\
\hline 4 & .15 & .38 & -.05 & .78 & -.03 & .87 \\
\hline 5 & .03 & .85 & -.07 & .68 & .11 & .54 \\
\hline 6 & .13 & .44 & -.02 & .90 & .11 & .54 \\
\hline 7 & -.14 & .43 & .08 & .66 & .13 & .46 \\
\hline 8 & -.08 & .65 & -.12 & .50 & -.31 & .07 \\
\hline 9 & .11 & .54 & -.06 & .74 & .07 & .68 \\
\hline 10 & .09 & .61 & .11 & .51 & .32 & .06 \\
\hline 11 & -.13 & .46 & .05 & .75 & .14 & .41 \\
\hline 12 & .05 & .79 & .07 & .68 & .07 & .66 \\
\hline 13 & .04 & .82 & .05 & .78 & .09 & .62 \\
\hline 14 & .13 & .44 & .02 & .93 & .02 & .92 \\
\hline 15 & .12 & .47 & -.05 & .75 & .07 & .67 \\
\hline 16 & -.08 & .65 & .05 & .79 & .47 & .00 \\
\hline 17 & .02 & .93 & .00 & .98 & -.13 & .44 \\
\hline
\end{tabular}

Tabla S3. Correlación de las métricas con la escala CDR-SB para las regiones de la red de control ejecutivo. Fuente: elaboración propia.

\begin{tabular}{ccccccc}
\hline \multirow{2}{*}{ ROI } & ALFF & \multicolumn{3}{c}{ fALFF } & \multicolumn{2}{c}{$\mathrm{PE}$} \\
\cline { 2 - 7 } & $\mathrm{r}$ & $\mathrm{P}$ & $\mathrm{R}$ & $\mathrm{p}$ & $\mathrm{r}$ & $\mathrm{p}$ \\
\hline 1 & .16 & .35 & -.16 & .35 & -.18 & .30 \\
2 & .08 & .62 & -.02 & .89 & -.28 & .10 \\
3 & .01 & .98 & -.05 & .79 & .13 & .45 \\
4 & .02 & .92 & -.01 & .95 & -.13 & .46 \\
5 & .16 & .35 & .07 & .68 & .07 & .71 \\
6 & .07 & .69 & -.15 & .38 & -.09 & .60 \\
7 & -.02 & .91 & .03 & .85 & .03 & .84 \\
8 & .03 & .86 & .13 & .44 & .02 & .90 \\
9 & .01 & .93 & .10 & .55 & -.03 & .85 \\
10 & .21 & .22 & -.06 & .73 & .31 & .06 \\
11 & .04 & .83 & .34 & .04 & .16 & .36 \\
\hline
\end{tabular}


Tabla S4. Correlación de las métricas con la escala MMSE para las regiones de la red de control ejecutivo.

\begin{tabular}{rrrrrrr}
\hline \multirow{2}{*}{ ROI } & \multicolumn{2}{c}{ ALFF } & \multicolumn{2}{c}{ fALFF } & \multicolumn{2}{c}{ PE } \\
\cline { 2 - 7 } & $\mathrm{r}$ & $\mathrm{p}$ & $\mathrm{R}$ & $\mathrm{p}$ & $\mathrm{r}$ & $\mathrm{p}$ \\
\hline 1 & .14 & .43 & .07 & .67 & .19 & .27 \\
2 & .04 & .82 & .21 & .22 & .15 & .37 \\
3 & .04 & .83 & -.04 & .83 & .17 & .33 \\
4 & -.18 & .28 & -.05 & .76 & -.04 & .80 \\
5 & -.10 & .54 & .02 & .93 & -.16 & .36 \\
6 & .11 & .52 & -.05 & .76 & .07 & .67 \\
7 & .09 & .62 & -.12 & .49 & .27 & .10 \\
8 & .02 & .91 & -.20 & .24 & .02 & .92 \\
9 & .14 & .42 & -.08 & .65 & .01 & .97 \\
10 & -.05 & .78 & .11 & .51 & .11 & .53 \\
11 & .03 & .84 & -.25 & .14 & .04 & .80 \\
\hline
\end{tabular}

